\title{
Un niño con traquioniquia de las 20 uñas
}

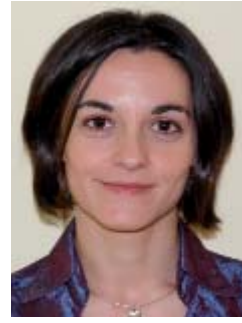

M. a Covadonga Martínez-González Dermatóloga. Práctica privada. Clínica de Cirugía Oral y Maxilofacial y Centro de Estética Facial Dr. Llorente. Oviedo. Asturias

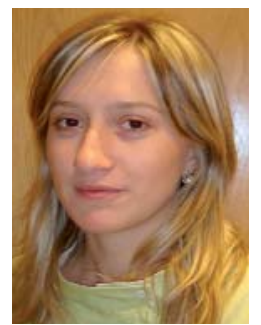

Noelia Marrón Álvarez Auxiliar de consulta y esteticista.

Clínica de Cirugía Oral y Maxilofacial y Centro de Estética Facial Dr. Llorente. Oviedo. Asturias
Mi paciente es un niño de 4 años que nos visitó por alteraciones en las 20 uñas. Entre sus antecedentes médicos, se encontraban bajo peso al nacer $(2,100 \mathrm{~kg})$ y amigdalectomía en el último mes, por problemas de apnea nocturna. La analítica de sangre poscirugía fue normal. No tomaba ninguna medicación y refería buen estado general. Acudió acompañado por su madre y ambos manifestaban cierta preocupación en la consulta. Como antecedentes familiares dermatológicos, destacaban lesiones que referían como «tipo psoriasis» en un tío materno, y la madre comentaba que padecía lesiones ocasionales en los codos, que no tenía en el momento de la consulta.
Había comenzado con lesiones de las 20 uñas hacía 6 meses, sin otra sintomatología más que roturas frecuentes. No refería placas de alopecia en cuero cabelludo ni lesiones cutáneas en otras zonas en ningún momento de su vida. En la exploración física, se observaban alteraciones ungueales consistentes en estriaciones longitudinales marcadas, que condicionaban una superficie ungueal áspera y rugosa, sin brillo y de aspecto descamativo (fig. 1). Las alteraciones eran más patentes en las uñas de los primeros dedos de manos y pies, y no se evidenciaban lesiones cutáneas en ningún otro lugar. En la dermatoscopia digital, se observaban importantes estriaciones longitudi-

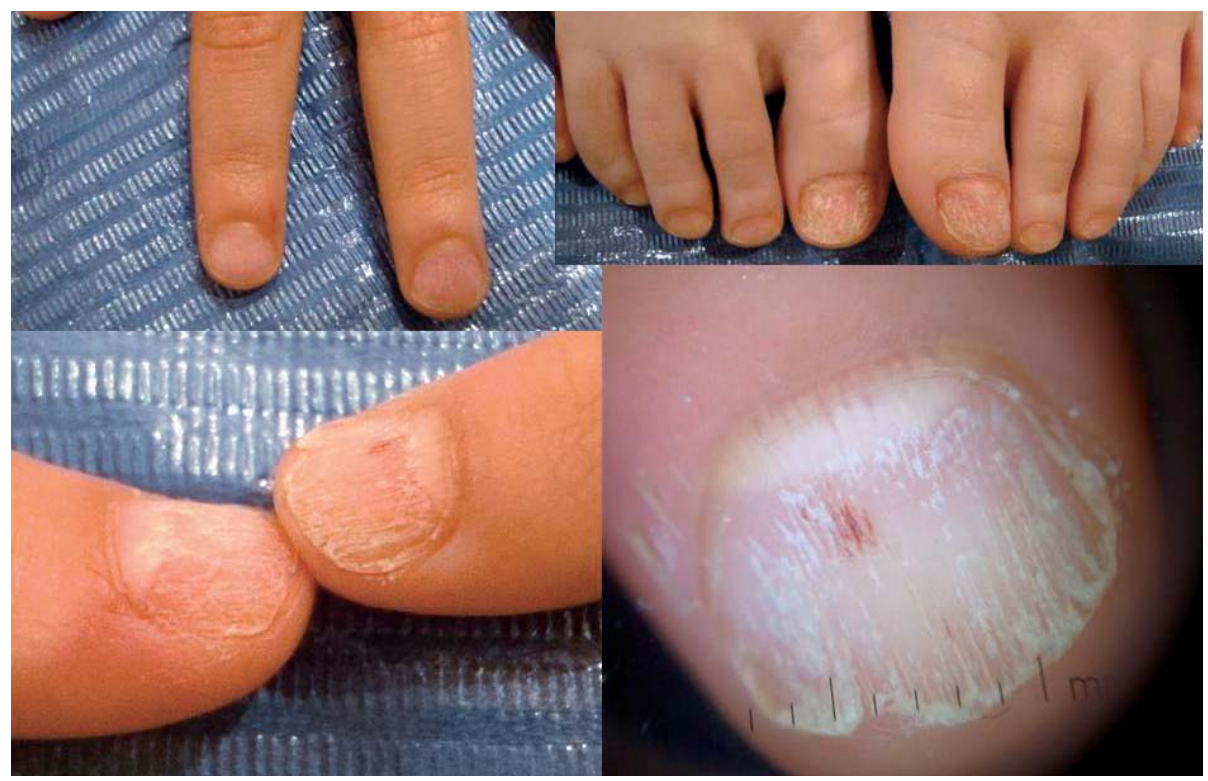

Figura 1. Estriaciones y surcos longitudinales propios de la traquioniquia que nuestro paciente presentaba en la primera visita. Se observa el detalle de la dermatoscopia digital de dichas lesiones. 
nales con onicólisis distal y alguna pequeña hemorragia en astilla (fig. 1).

Se decidió no realizar biopsia por el riesgo de distrofia ungueal permanente y se consideró el caso compatible con traquioniquia de las 20 uñas.

Su madre comentaba que se trataba de un niño cariñoso y muy sensible, con tendencia a padecer de estrés y en concreto, en el último año, por el destino laboral de su padre en otra ciudad.

Hacía 1 año que su padre había sido trasladado a otra ciudad por conveniencia laboral. Solo se veían desde entonces los fines de semana. $\mathrm{Su}$ madre comentaba que esta situación fue bien llevada emocionalmente por el niño los primeros 3 meses. A partir de entonces, el niño comenzó a notar más la carencia de su padre y no aceptaba bien la situación de separación familiar entre semana y las despedidas de fin de semana.

Se tranquilizó al paciente y a su madre, comentando la benignidad habitual del curso de la enfermedad y la posible relación con factores de estrés emocional asociados. Se pautó tratamiento con crema de urea al $40 \%$ y propionato de clobetasol en crema, tres veces por semana. El seguimiento a los 4 meses fue muy favorable (fig. 2).
Se comentó con ellos que, ante la aparición de otra nueva manifestación dermatológica, se revaloraría el caso.

La traquioniquia es un trastorno de la lámina ungueal que se caracteriza por una superficie ungueal áspera, rugosa, con marcado pitting y estriaciones longitudinales (aspecto de haber sido tratada con lija) ${ }^{1}$. El síndrome de las 20 uñas presenta estas alteraciones en todas las uñas y puede aparecer como signo aislado o asociado con otras enfermedades dermatológicas: alopecia areata, liquen plano y psoriasis (entre las más características) ${ }^{2}$. Está ampliamente documentado que los brotes de estas últimas enfermedades dermatológicas podrían aparecer en relación con situaciones de estrés emocional ${ }^{3,4,5}$.

La traquioniquia de las 20 uñas, también llamada «distrofia de las 20 uñas», es una entidad que cobra especial interés desde hace unos 30 años $^{6}$. El término describe una rugosidad característica de la superficie de la lámina ungueal de todas o la mayor parte de las uñas, de forma habitualmente bilateral y simétrica, que cursa normalmente sin dolor ni otras manifestaciones clínicas, excepto las roturas ungueales frecuentes que la propia dis-

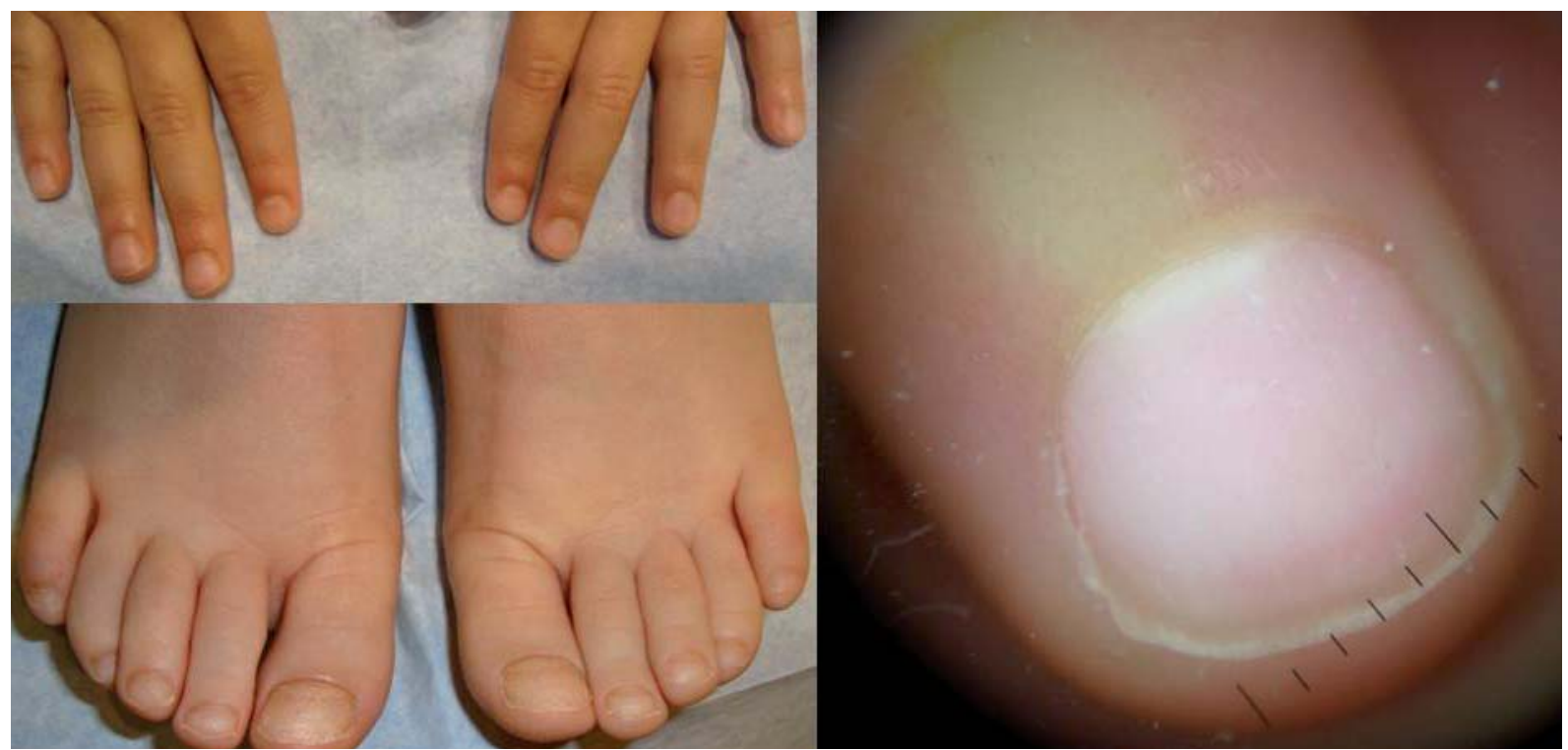

Figura 2. Se observa mejoría clínica evidente a los 4 meses de la primera visita, una vez efectuado el diagnóstico y planteado el tratamiento. 
trofia conlleva. Se trata de una enfermedad inflamatoria benigna del aparato ungueal ${ }^{6,7}$. Se han descrito casos de presencia al nacimiento, con progresión lenta, e incluso casos familiares con herencia autosómica dominante, pero en la mayor parte de los casos, se trata de un proceso adquirido con evolución autolimitada ${ }^{1}$. Afecta fundamentalmente a niños o adolescentes, con una incidencia similar en ambos sexos, y es menos habitual en adultos $^{1,2}$. Puede aparecer de forma clínicamente aislada en muchos casos, y se considera una entidad idiopática. También puede aparecer en el contexto o asociada a otras enfermedades o dermatosis, entre las cuales se han descrito de forma más frecuente la alopecia areata (especialmente en sus formas más severas) y también la psoriasis y el liquen plano. Otras enfermedades documentadas en asociación han sido: dermatitis atópica, ictiosis vulgar, vitíligo, incontinencia de pigmento, pénfigo vulgar, sarcoidosis, síndrome de Sézary, púrpura trombocitopénica, anemias hemolíticas, poliendocrinopatías y déficit selectivo de $\operatorname{IgA}^{1,2,6-9}$. Ante la presencia de traquioniquia, deben descartarse clínicamente estas asociaciones ${ }^{1}$.

El diagnóstico es fundamentalmente clínico, ya que los cambios ungueales son muy representati$\operatorname{vos}^{2}$. Este diagnóstico podría completarse con una biopsia longitudinal de la lámina ungueal. Esto último no siempre se realiza, por el riesgo que conlleva de distrofia ungueal permanente, hasta en un tercio de los $\operatorname{casos}^{8}$. Cuando se practica en ocasiones pueden encontrarse hallazgos histopatológicos específicos de psoriasis o liquen plano (desde casos aislados hasta en un 52\% para el conjunto de estas dos entidades, según las series estudiadas) $)^{2,7,8}$. Pero lo más habitual es encontrar cambios espongióticos inespecíficos ${ }^{7,8}$.

La traquioniquia suele mejorar espontáneamente con los años (según algunas series, el 50\% presenta una resolución total o una gran mejoría en los primeros 6 años de seguimiento, sin tratamiento), por lo que no siempre requiere intervención terapéutica ${ }^{10}$. Esta puede reservarse para casos que conlleven una preocupación excesiva asociada, especialmente por parte de los padres. Entre los tratamientos descritos destacan: corticoides tópicos o sistémicos, biotina por vía oral, PUVA, tazaroteno, apósitos ungueales (con ácido láctico, dióxido de silicio, acetilacetonato de aluminio y copolímero de ácido acrílico acetato de vinilo) $)^{1,2}$. Es importante el seguimiento para ver si, con el tiempo, se diagnostica alguna dermatosis y, en este caso, realizar el tratamiento de la misma. De la misma forma, es importante tranquilizar al paciente y la familia, incidiendo en la benignidad y la autorresolución del proceso pasados unos años.

En nuestro paciente, de forma particular, nos parece que la situación de estrés emocional que estaba viviendo le podría haber condicionado un brote de dermatosis inflamatoria, que en su caso se localiza en la matriz ungueal y no en el resto del tegumento cutáneo hasta la fecha, por lo que la clínica se evidencia en la superficie de la lámina ungueal y se presenta como un caso de traquioniquia de las 20 uñas.

\section{BIBLIOGRAFÍA}

1. Arias-Santiago $S$, Fernández-Pugnaire MA, Husein El-Ahmed H, Girón-Prieto MS, Naranjo Sintes R. A 9 year-old child with trachyonychia: a good response with nail plate dressings. An Pediatr. 2009;71;5:476-7.

2. Sehgal VN. Twenty nail dystrophy trachyonichya: an overview. J Dermatol. 2007;34:361-6.

3. Díaz-Atienza F, Gurpegui M. Enviromental stress but not subjective distress in children or adolescent with alopecia areata. J Psychosom Res. 2011;71:102-7.

4. Heller MM, Lee ES, Koo JY. Stress as an influencing factor in psoriasis. Skin Therapy Lett. 2011;16:1-4.

5. Manolache L, Seceleanu-Petrescu D, Benea V. Lichen planus patients and stressful events. J Eur Acad Dermatol Venereol. 2008;22:437-41

6. Samman PD. Trachyonichya (rough nails). Br J Dermatol. 1979;101:701-5.

7. Grover C, Khandpur S, Reddy BS, Chaturvedy KU. Longitudinal nail biopsy: utility in 20-nail dystrophy. Dermatol Surg. 2003; 29:1125-9.

8. Tosti A, Bardazzi F, Piraccini BM, Fanti PA. Idiopathic trachyonychia (twenty-nail dystrophy): a pathological study of 23 patients. Br J Dermatol. 1994;131:866-72.

9. Blanco FP, Scher RK. Trachyonychia: case report and review of the literature. J Drugs Dermatol. 2006;5:469-72.

10. Sakata S, Howard A, Tosti A, Sinclair R. Follow up of 12 patients with trachyonychia. Australas J Dermatol. 2006;47:166-8. 